\title{
Preparation and Characterization of Silica-coated Gold Nanoflowers (AuNFs) with Raman Dye Encoding
}

\author{
Jihye Yoo and Sang-Wha Lee* \\ Department of Chemical \& Biochemical Engineering, Gachon University, Seongnam 461-701, Korea \\ "E-mail: lswha@gachon.ac.kr \\ Received February 27, 2014, Accepted May 27, 2014
}

\begin{abstract}
Flower-like Au nanoparticles, so-called Au nanoflowers (AuNFs), were synthesized by simply adding ascorbic acid to a gold acid solution in the presence of a chitosan biopolymer. The chitosan-entangled AuNFs exhibited strong plasmon absorption in the near-infrared (NIR) wavelength due to the aggregation of primary Au nanoparticles. The chitosan-entangled AuNFs were preferentially adsorbed by Raman-active 2-chlorothiophenol (CTP) molecules, and the CTP-encoded AuNFs (AuNF-CTPs) were subsequently coated with a thin silica layer by a sol-gel reaction with Si alkoxides. The silica-coated AuNFs (AuNF-CTPs@silica) exhibited the distinct Raman signals of adsorbed CTP molecules, as a potential nanoprobe with surface-enhanced Raman scattering (SERS).
\end{abstract}

Key Words : Gold nanoflowers, Chitosan, SERS, Silica layer

\section{Introduction}

Since Raman spectroscopy was first successfully applied to cell bioanalysis in the 1990s, surface-enhanced Raman scattering (SERS) has become a powerful analytical tool for the ultrasensitive and highly-resoved identification of biomolecules due to the enormous amplification $\left(10^{6}-10^{12}\right)$ of Raman scattering at so-called "hot spots". 1,2 The fabrication of SERS-active substrates is currently focused on Raman dye-encoded nanoparticles because of their applications to biolabeling, cell imaging, and even cell targeting. ${ }^{3-5}$ For a facile biomedical application, SERS nanoprobe must have biocompatible and tailorable surface morphology. Recently, increasing attention has been directed for wrapping a SERS nanoprobe with a coating layer which can protect from oxidative environment. ${ }^{6,7}$ Furthermore, it would be very beneficial if the coating layer could provide conjugation with functional groups of various bioagents.

Gold nanoparticles with edged shapes (e.g., cube, triangle, star, multipod, flower-like, etc.) are attractive because of the localization of intense electromagnetic fields around the sharp and/or interstitial sites. ${ }^{8,9}$ Flower-like Au nanoparticles, i.e., Au nanoflowers (AuNFs), have recently been reported as a highly effective SERS substrate. ${ }^{10-12}$ Wang et al. prepared regular array of gold nanoflowers using electrochemical deposition on ITO conductive substrate and applied them as SERS substrates to probe rhodamine $6 \mathrm{G} .^{13}$ Pradeep et al. also prepared $\mathrm{Au} / \mathrm{Ag}$ mesoflowers as a SERS substrate which were able to detect probe molecules sensitively. ${ }^{14}$ However, it is rarely found that AuNFs encoded with strong Raman dyes have been applied as a SERS nanoprobe. ${ }^{3}$

In this work, we prepared flower-like Au nanoparticles using ascorbic acid agents in the presence of chitosan, and the synthesized AuNFs were adsorbed by Raman-active 2chlorothiophenol (CTP) molecules. Importantly, CTP-encoded
AuNFs were subsequently coated by a thin silica layer via a sol-gel reaction, in order to provide protection and conjugation with functional groups. The as-prepared samples were characterized by scanning electron microscopy (SEM), transmission electron microscopy (TEM), dynamic light scattering (DLS), UV-vis spectroscopy, and micro-Raman spectroscopy.

\section{Experimental}

Chemical Materials. The following reagents were purchased from Aldrich Chemical Co.: gold chloride hydrate $\left(\mathrm{HAuCl}_{4}\right)$, L-ascorbic acid $(99.0 \%)$, tetraethyl orthosilicate (TEOS, 99.999\%), ammonia hydroxide $\left(\mathrm{NH}_{4} \mathrm{OH}, 28 \mathrm{wt} \%\right.$ ), ethanol $\left(\mathrm{C}_{2} \mathrm{H}_{5} \mathrm{OH}, 99.5 \%\right)$, and chitosan $(50 \mathrm{kDa})$ with a deacetylation degree above $85 \%$. Acetic acid (99.0\%) was purchased from Duksan Pure Chemical, Ltd. and deionized water (HPLC grade) was purchased from J.T. Baker.

Au Nanoflowers (AuNFs). Chitosan was dissolved in $10 \mathrm{~mL}$ of $1.0 \% \mathrm{v} / \mathrm{v}$ acetic acid solution by sonication until transparent solution was obtained. $700 \mu \mathrm{L}$ of $1.0 \mathrm{wt} \%$ $\mathrm{HAuCl}_{4}$ was added to $3 \mathrm{~mL}$ of the chitosan solution under continuous stirring for $30 \mathrm{~min}$. Subsequently, $600 \mu \mathrm{L}$ of ascorbic acid $(0.1 \mathrm{~g}$ ascorbic acid in $10 \mathrm{~mL}$ HPLC water) was quickly added to the mixed solution with continuous stirring for $30 \mathrm{~min}$. The amine groups of chitosan induced the electrostatic interaction with negatively charged Au NPs and the scrolling capacity of vicinal chitosan chains triggered the agglomerization of primary $\mathrm{Au}$ NPs, consequently leading to the formation of flower-like Au NPs. ${ }^{10}$ The resulting AuNFs were purified by centrifuging them three times and re-suspending them in $3 \mathrm{~mL}$ of HPLC water to remove the residual components.

Silica-coated AuNFs. For the SERS measurements, an aliquot of 2-chlorothiophenol (CTP, $10^{-3} \mathrm{M}$ ) was added into 
the AuNFs solution $(4 \mathrm{~mL})$ and gently stirred for $4 \mathrm{~h}$ to obtain the equilibrium state of CTP-adsorbed AuNFs (CTPAuNFs). Subsequently, CTP-AuNFs were coated with silica layer according to the modified Stöber method. ${ }^{15} 15 \mathrm{~mL}$ of isopropanol was mixed with $2 \mathrm{~mL}$ of AuNFs solution, and then $150 \mu \mathrm{L}$ of ammonium solution (28 wt \%) was injected into the mixed solution under constant stirring for $10 \mathrm{~min}$. The sol-gel reaction proceeded for $6 \mathrm{~h}$ by the injection of TEOS solution, which was prepared by mixing $15 \mu \mathrm{L}$ of TEOS (99\%) with $10 \mathrm{~mL}$ of HPLC water for $10 \mathrm{~s}$. The final products were centrifuged for $20 \mathrm{~min}$ at $7000 \mathrm{rpm}$ and the purified products were re-suspended in $3 \mathrm{~mL}$ of HPLC water for a further analysis.

Instrumental Analysis. The IR spectra were recorded with a resolution of $2 \mathrm{~cm}^{-1}$ using a Fourier transform infrared spectrometer (FTIR, VERTEX 70, Bruker). The distribution of particle size was measured at room temperature using an intensity-based dynamic light scattering (DLS) method. The surface morphology of the particles was analyzed by SEM (Hitachi S-4700) operating at $15 \mathrm{kV}$ and TEM (Hitachi H7600) operating at the acceleration voltage of $200 \mathrm{kV}$. UVvis absorption spectra (Model: HP 8453) was measured in the wavelength region of 200-1000 $\mathrm{nm}$. The Raman spectrograph (ANDOR Monora500i) employed a $1200 \mathrm{~g} / \mathrm{mm}$ grating under a laser excitation at $632.8 \mathrm{~nm}$ for $5 \mathrm{~s}$.

\section{Results and Discussion}

Scheme 1 shows the fabrication process for silica-coated Au nanoflowers encoded with Raman dyes (AuNF-CTP@ silica). First, flower-like Au nanoparticles (AuNFs) were prepared by adding ascorbic acid into a gold acid solution in the presence of chitosan biopolymers. The scrolling capacity of the chitosan chains induced the agglomeration of the primary $\mathrm{Au}$ nanoparticles, thus forming flower-like AuNFs. The chitosan-entangled AuNFs were adsorbed by Raman dyes and the AuNF-Raman dye complex was finally coated with thin silica layer by the sol-gel reaction with Si precursor of TEOS.

The AuNFs were prepared by mixing a gold acid solution with ascorbic acid in the presence of chitosan biopolymers with different concentrations. According to Figures 1(a-d), the size of the AuNFs decreased with increasing chitosan concentration from $0.01 \mathrm{wt} \%$ to $0.4 \mathrm{wt} \%$. At a low chitosan concentration $(0.01 \mathrm{wt} \%)$, the primary Au NPs were not fully entangled with chitosan chains and the agglomeration of primary Au NPs became more dominant, consequently forming large AuNFs. ${ }^{10}$ At the intermediate chitosan con-

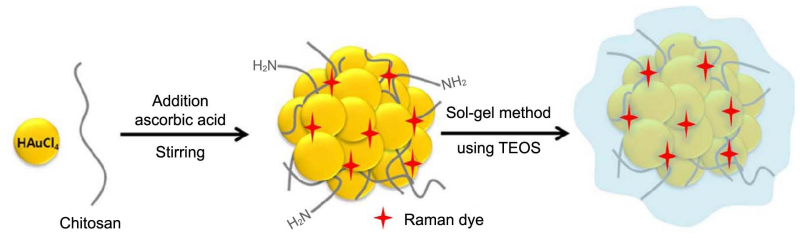

Scheme 1. Schematic diagram of synthesizing silica-coated AuNFs encoded with Raman dyes. centrations (0.1-0.2 wt \%), the obtained particles exhibited the flower-like Au NPs (AuNFs) and the sizes of which were decreased as compared to AuNFs preapred at low chitosan concetration $(0.01 \mathrm{wt} \%)$. At a high chitosan concentration $(0.4 \mathrm{wt} \%)$, the primary Au NPs were immediately entangled by the chitosan chains, consequently forming the small AuNFs.

The corresponding UV-vis spectra of the AuNFs (0.4 wt \% of chitosan) exhibited a broad absorption band centered at $658 \mathrm{~nm}$, whereas Au nanoparticles prepared only by ascorbic acid exhibited the characteristic absorption peak at $536 \mathrm{~nm}$ (see Figure S1 in supplemental materials). The significant red-shift of the localized surface plasmon resonances was attributed to the flower-like aggregation of the primary $\mathrm{Au}$ NPs. ${ }^{3,16}$ The AuNFs prepared with a chitosan concentration of $0.2 \mathrm{wt} \%$ were subsequently coated by a thin silica layer through a sol-gel reaction between the AuNFs and TEOS. According to DLS analysis, the size of the silica-coated AuNFs increased 1.5-2 times as compared to pristine AuNFs (data not shown) even though the SEM images shown in Figure S2 (in supporting information) did not show a noticeable size difference between silica-coated AuNFs.
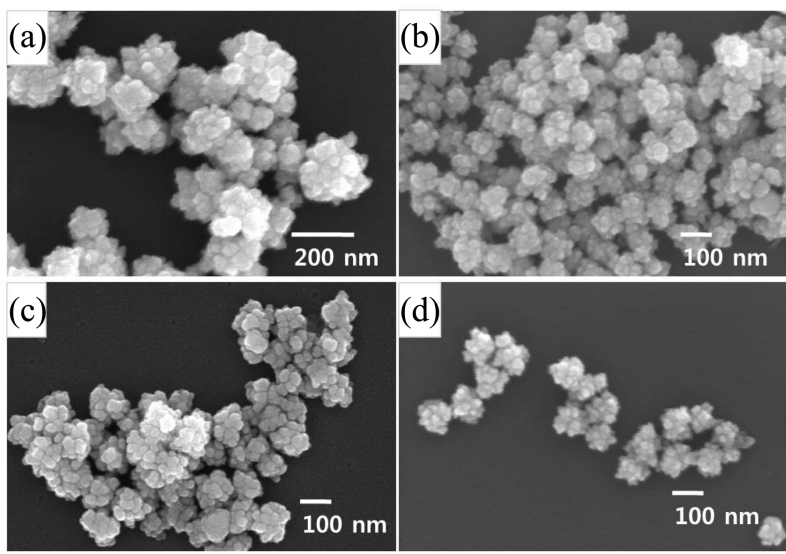

Figure 1. SEM images of AuNFs prepared by adding $600 \mu \mathrm{L}$ of ascorbic acid and $150 \mu \mathrm{L}$ of $\mathrm{NH}_{4} \mathrm{OH}$ in the presence of chitosan biopolymers: (a) 0.01 , (b) 0.1 , (c) 0.2 , and (d) $0.4 \mathrm{wt} \%$ of chitosan.

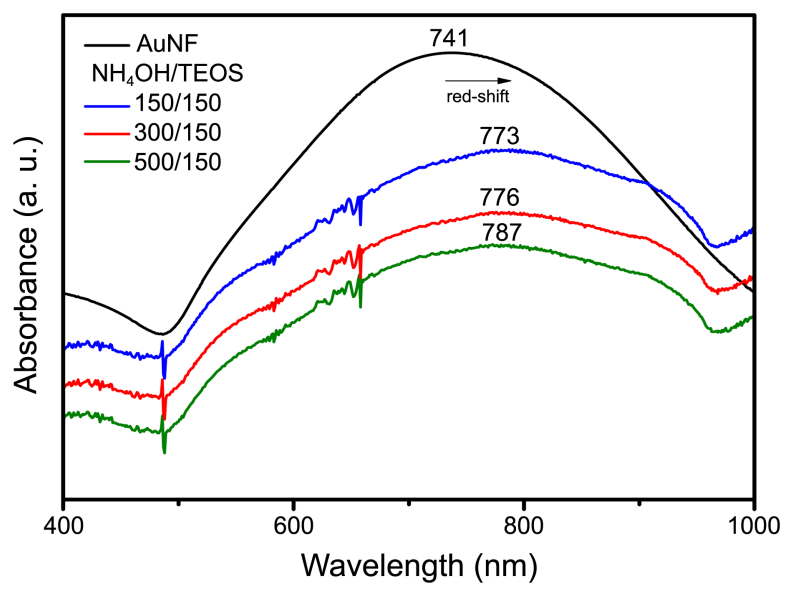

Figure 2. UV-vis spectra of silica-coated AuNFs prepared by adding $150 \mu \mathrm{L}$ of TEOS and different amounts of $\mathrm{NH}_{4} \mathrm{OH}$ : (a) $0 \mu \mathrm{L}$, (b) $150 \mu \mathrm{L}$, (c) $300 \mu \mathrm{L}$, (d) $500 \mu \mathrm{L}$. 
Figure 2 shows the UV-vis spectra of pristine AuNFs ( 0.2 wt $\%$ of chitosan) and silica-coated AuNFs prepared at different amounts of $\mathrm{NH}_{4} \mathrm{OH}$. The silica-coated AuNFs exhibited a red-shift of the maximal absorbance peak from $741 \mathrm{~nm}$ to $\sim 773-787 \mathrm{~nm}$. The red-shift of the absorbance peak indicates the change of the surrounding refractive index from water (1.33) to a silica layer (1.57). ${ }^{17}$ The increase of $\mathrm{NH}_{4} \mathrm{OH}$ decreased the intensity of the UV-vis absorption peak, probably due to the aggregation of the silica-coated AuNFs.

Figure 3 shows the change of zeta potentials of AuNFs according to the surface modifications such as Raman dyeencoding and the silica coating. The chitosan-entangled AuNFs exhibited a positive zeta potential, as expected from the positive amine groups of chitosan chains. ${ }^{18}$ The Raman dye-encoded AuNFs exhibited a almost zerol value of zeta potential, $-0.45 \mathrm{mV}$, probably due to the screening effects of the adsorbed CTP on the AuNFs. However, the zeta potential of the silica-coated AuNFs exhibited a distinctly negative zeta potential, $-20.85 \mathrm{mV}$, indicating the presence of silica layer on the AuNFs. ${ }^{19}$ As a reference, Au NPs prepared by ascorbic acid without chitosan exhibited a negative zeta potential, $-5.72 \mathrm{mV}$, due to the negative surface charges of

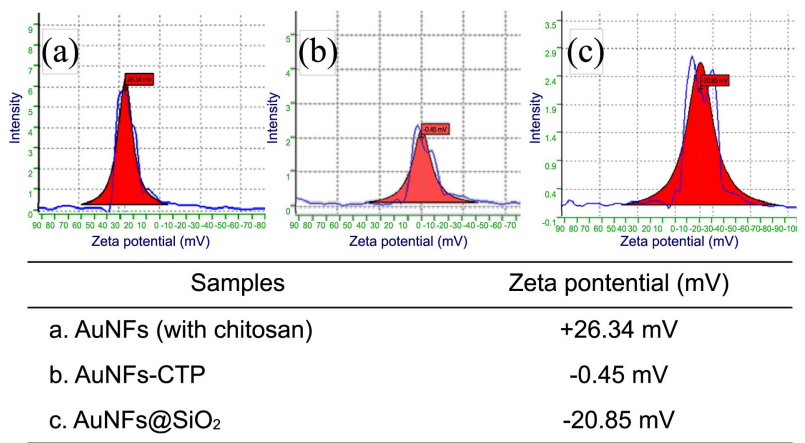

Figure 3. Zeta potentials of AuNFs, AuNF-CTPs, and AuNFCTPs@SiO $\mathrm{SiO}_{2}$.

(a)

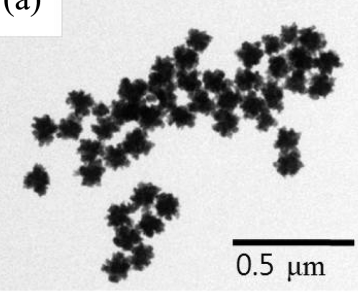

(c)

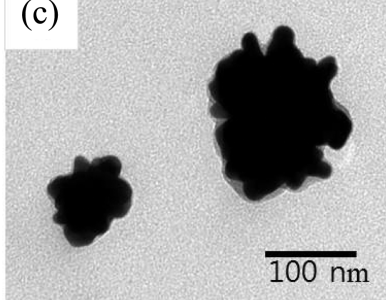

Figure 4. TEM images of (a) pristine AuNFs, (b) magnified image of (a), (c) silica-coated AuNFs (AuNF-CTPs@SiO $\mathrm{Si}_{2}$ ), and (d) SAED patterns of AuNFs.

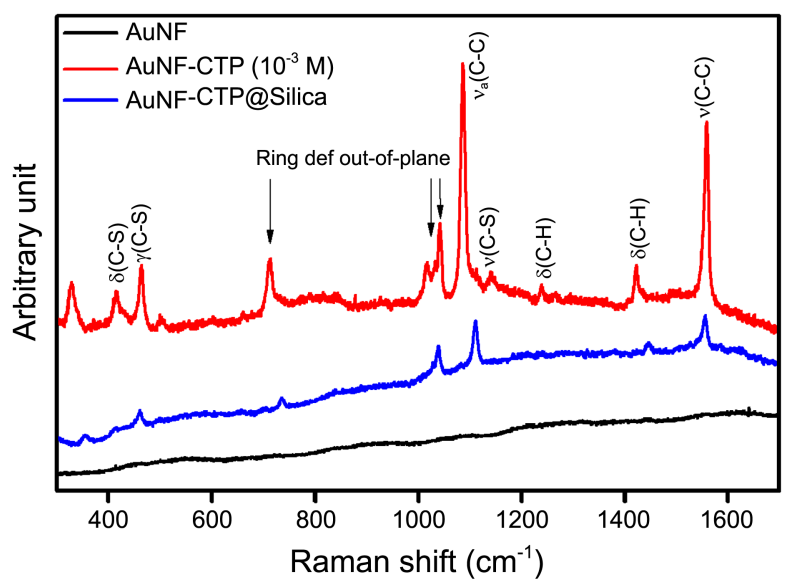

Figure 5. Raman signals of AuNFs, 2-chlorothiolphenol (CTP, $10^{-3} \mathrm{M}$ ) adsorbed on AuNFs (AuNF-CTPs), and silica-coated AuNFs (AuNF-CTPs@SiO $\mathrm{SiO}_{2}$ ).

the primary Au NPs.

Figures 4(a-c) show TEM images of the pristine AuNFs and the silica-coated AuNFs. Figure 4(a) shows the size uniformity of as-prepared samples. The morphology of the flower-like Au NPs was clearly observed from the magnified TEM image shown in Figure 4(b). The magnified TEM image exhibits multipod and spike-shaped tips ranging in size from $10 \mathrm{~nm}$ to $20 \mathrm{~nm}$. The anisotropic AuNFs formed by the aggregated primary $\mathrm{Au}$ NPs provided a surface morphology consisting of junctions, edges, and corners, which could act as "hot-spots" in SERS measurements. 5,14 According to Figure 4(c), the silica-coated AuNFs exhibited a distinct contrast between the thin silica overlayer and the AuNF core. The selected area electron diffraction (SAED) pattern indicated the polycrystalline structure of the AuNFs, as shown in Figure 4(d). ${ }^{9}$

Figure 5 shows the Raman spectra of the pristine AuNFs, the AuNF-CTPs, and the AuNF-CTPs@silica. No clear Raman signals were observed from the chitosan molecules entangled with AuNFs. On the other hand, AuNF-CTPs and AuNF-CTPs@silica exhibited distinct Raman signals of adsorbed CTP molecules owing to the highly anisotropic nanostructures of the AuNFs, which can create many hot spots for a localized near-field enhancement effect. ${ }^{3,5}$ The largest Stokes-shifted vibration was observed at $1570 \mathrm{~cm}^{-1}$, which was attributed to the $v(\mathrm{C}-\mathrm{C})$ vibration. The Raman peaks observed at $1020 \mathrm{~cm}^{-1}$ and $690 \mathrm{~cm}^{-1}$ were attributed to ring def in-plane and out-of-plane, respectively. The Raman peaks observed at $460 \mathrm{~cm}^{-1}$ for both samples were attributed to the $\gamma(\mathrm{C}-\mathrm{S})$ vibration of thiophenol molecules. ${ }^{20}$ The Raman signals of AuNF-CTPs@silica were fairly reduced as compared to those of AuNF-CTPs. The low intensity of SERS may come from the aggregation of AuNF-CTPs@silica and/or interference of the silica shell over encoded Raman dyes. $^{21}$

\section{Conclusion}

In this work, we prepared chitosan-entangled flower-like 
Au nanoparticles (gold nanoflowers, AuNFs) using ascorbic reducing agents. The AuNFs exhibited strong plasmon absorption in the NIR (Near infrared) region caused by the aggregation of primary Au NPs. The chitosan-entangled AuNFs were adsorbed by Raman-active CTP molecules, and the resulting CTP-encoded AuNFs (AuNF-CTPs) were coated with a thin silica layer via a sol-gel reaction with $\mathrm{Si}$ alkoxides. The AuNF-CTPs and silica-coated AuNFs (AuNF-CTPs@silica) exhibited highly enhnaced Raman signals of adsorbed CTP molecules. Thus, silica-coated AuNFs with Raman dye encoding can be employed as a SERS nanoprobe with versitile conjugation.

Acknowledgments. This work was supported by the GRRC program of Gyeonggi province [GRRC Gachon 2014-B04, Development of nanomaterials for biomedical sensing applications].

\section{References}

1. Xie, W.; Su, L.; Shen, A.; Materny, A.; Hu, J. J. Raman Spec. 2011, $42,1248$.

2. Chen, C.; He. X.; Gao, L.; Ma, N. ACS Appl. Mater. Interfaces 2013, $5,1149$.

3. Xie, J. P.; Zhang, Q. B.; Lee, J. Y. ACS Nano 2008, 2, 2473.

4. Li, J.; Wu, J.; Zhang, X.; Liu, Y.; Zhou, D.; Sun, H.; Zhang, H.;
Yang, B. J. Phys. Chem. C 2011, 115, 3630.

5. Jiang, Y.; Wu, X.; Li, Q.; Li, J.; Xu, D. Nanotechnology 2011, 22, 385601.

6. Tan, X. B.; Wang, Z. Y.; Yang, J. Nanotechnology 2009, 20, 445102.

7. Woo, M.; Lee, S.; Kim, G.; Baek, J.; Noh, M. S.; Kim, J. E.; Park, S. J.; Minai-Tehrani, A.; Park, S.; Seo, Y. T.; Kim, Y.; Lee, Y.; Jeong, D. H.; Cho, M. Anal. Chem. 2009, 81, 1008.

8. Kumar, B.; Raj, C. R. Chem. Mater. 2008, $20,3546$.

9. Ren, Y.; Xu, C.; Wu, M.; Niu, M.; Fang, Y. Colloids and Surfaces A: Physicochemical and Engineering Aspects 2011, 380, 222.

10. Nhung, T. T.; Lee, S. J. Cryst. Growth 2013, 373, 132.

11. Mohanty, A.; Garg, N.; Jin, R. Angew. Chem. Int. Ed. 2010, 49, 4962.

12. Wang, Z. D.; Zhang, J. Q.; Ekaman, J. M.; Kenis, P. J. A.; Lu, Y. Nano Lett. 2010, 10, 1886.

13. Wang, J.; Duan, G.; Li, Y.; Liu, G.; Dai, Z.; Zhang, H.; Cai, W. Langmuir 2013, 29, 3512.

14. Sajanlal, P. R.; Pradeep, T. Langmuir 2010, 26, 8901.

15. Park, S.; Lee, J.; Haam, S.; Lee, S. Bull. Korean Chem. Soc. 2009, 30,869 .

16. Huang, L.; Wang, M.; Zhang, Y.; Guo, Z. R.; Sun, J. F.; Gu, N. J. Phys. Chem. C 2007, 111, 16154.

17. Doering, W. E.; Nie, S. Anal. Chem. 2003, 75, 6171.

18. Jang, M.; Nah, J. Bull. Korean Chem. Soc. 2003, 24, 1303.

19. Park, S.; Lee, J.; Haam, S.; Lee, S. Bull. Korean Chem. Soc. 2009, 30,869 .

20. Mahmoud, M. A.; El-Sayed, M. A. Nano Lett. 2009, 9, 3025.

21. Mulvaney, S. P.; Musick, M. D.; Keating, C. D.; Natan, M. J. Langmuir 2003, 19, 4784. 